\title{
The $203 \mathrm{kbp}$ Mitochondrial Genome of the Phytopathogenic Fungus Sclerotinia borealis Reveals Multiple Invasions of Introns and Genomic Duplications
}

\author{
Andrey V. Mardanov, Alexey V. Beletsky, Vitaly V. Kadnikov, Alexander N. Ignatov, Nikolai V. Ravin*
}

Centre "Bioengineering", Russian Academy of Sciences, Moscow, Russia

\begin{abstract}
Here we report the complete sequence of the mitochondrial $(\mathrm{mt})$ genome of the necrotrophic phytopathogenic fungus Sclerotinia borealis, a member of the order Helotiales of Ascomycetes. The 203,051 bp long mtDNA of $S$. borealis represents one of the largest sequenced fungal $\mathrm{mt}$ genomes. The large size is mostly determined by the presence of mobile genetic elements, which include 61 introns. Introns contain a total of 125,394 bp, are scattered throughout the genome, and are found in 12 protein-coding genes and in the ribosomal RNA genes. Most introns contain complete or truncated ORFs that are related to homing endonucleases of the LAGLIDADG and GIY-YIG families. Integrations of mobile elements are also evidenced by the presence of two regions similar to fragments of inverton-like plasmids. Although duplications of some short genome regions, resulting in the appearance of truncated extra copies of genes, did occur, we found no evidences of extensive accumulation of repeat sequences accounting for mitochondrial genome size expansion in some other fungi. Comparisons of mtDNA of $S$. borealis with other members of the order Helotiales reveal considerable gene order conservation and a dynamic pattern of intron acquisition and loss during evolution. Our data are consistent with the hypothesis that horizontal DNA transfer has played a significant role in the evolution and size expansion of the S. borealis mt genome.
\end{abstract}

Citation: Mardanov AV, Beletsky AV, Kadnikov VV, Ignatov AN, Ravin NV (2014) The 203 kbp Mitochondrial Genome of the Phytopathogenic Fungus Sclerotinia borealis Reveals Multiple Invasions of Introns and Genomic Duplications. PLoS ONE 9(9): e107536. doi:10.1371/journal.pone.0107536

Editor: Alexander F. Palazzo, University of Toronto, Canada

Received February 25, 2014; Accepted August 19, 2014; Published September 12, 2014

Copyright: (C) 2014 Mardanov et al. This is an open-access article distributed under the terms of the Creative Commons Attribution License, which permits unrestricted use, distribution, and reproduction in any medium, provided the original author and source are credited.

Funding: This work was supported by the Russian Foundation for Basic Research (grant 12-04-33122) and by the "Molecular and Cellular Biology" program of the Russian Academy of Science. The funders had no role in study design, data collection and analysis, decision to publish, or preparation of the manuscript.

Competing Interests: The authors have declared that no competing interests exist.

*Email: nravin@biengi.ac.ru

\section{Introduction}

The phytopathogenic fungus Sclerotinia borealis Bubak \& Vleugel, which is an ascomycete belonging to the family Sclerotiniaceae of the order Helotiales, has a broad host range and causes diseases in at least 17 plant genera from the families Alliaceae, Asteraceae, Brassicaceae, Campanulaceae, Fabaceae, Iridaceae, Pinaceae, and Poaceae (notably wheat and corn). S. borealis is a psychrophilic necrotrophic fungus with an optimum growth temperature between $4^{\circ} \mathrm{C}$ and $10^{\circ} \mathrm{C}$ [1]. The main specific feature of the disease called "snow mould" is a white mycelium and sclerotia growth on dead plant tissues [2]. S. borealis is distributed mostly in northern regions (Japan, North America, Scandinavia, and Russia). However, the biology of S. borealis and its phylogenetic relationships to the other species of Sclerotiniaceae are still poorly understood.

Mitochondrial $(\mathrm{mt})$ genomes have been successfully used in evolutionary biology and systematic studies [3], [4], since they evolve faster than nuclear genomes [5], [6]. Fungal mt genomes range in size from about $19 \mathrm{kbp}$ (Hanseniaspora uvarum) to 235,849 bp for Rhizoctonia solani AG-3 strain RhslAP [7], and usually contain 14 genes that encode oxidative phosphorylation system proteins, the large $(r n l)$ and small $(r n s)$ ribosomal RNA subunits, and a fairly constant set of tRNAs genes [8], [9]. Besides this core set of genes, a varying number of introns, often including
GIY-YIG or LAGLIDADG endonuclease genes, have been reported [10], [11], [12], [13].

In recent years, the number of complete filamentous fungal $\mathrm{mt}$ genome sequences has significantly increased [14], [15], facilitating evolutionary and systematic studies [16], [17], [18], [19], [20], [21], [22]. Currently more than 100 complete fungal mitochondrial genomes are available, but only nine represent the order Helotiales of ascomycetes (Phialocephala subalpina, Sclerotinia sclerotiorum, Botrytis cinerea (teleomorph Botryotinia fuckeliana), Glarea lozoyensis, Marssonina brunnea, and four Rhynchosporium species) [16], [21], [22]. Only two mitochondrial genomes belong to members of the Sclerotiniaceae family, B. fuckeliana and $S$. sclerotiorum.

Comparative analysis of mtDNAs of three helotialean species, $P$. subalpina, B. fuckeliana, and $S$. sclerotiorum, revealed conservation of core $\mathrm{mt}$ genes, but high variability of mtDNA size, which ranged from 43,742 bp (P. subalpina) to $128,852 \mathrm{bp}$ (S. sclerotiorum [16]. Considerable variations of mtDNA size were observed even among species of a single genus, Rhynchosporium, from 49,539 bp (Rhynchosporium orthosporum) to 69,581 bp (Rhynchosporium commune) [22]. The large size variations of $\mathrm{mt}$ genomes between different fungal species mainly result from the presence or absence of large intronic and intergenic sequences [23], [24], [25], [26]. How the origin and direction of intron acquisition and loss are determined remains poorly understood. 
One hypothesis suggests that introns were abundant in the ancestral mt genes, but have subsequently been lost in most lineages [27]. Although this hypothesis is currently being discussed [28], both intron loss and gain events are required to explain the uneven distribution of introns across even rather closely related lineages.

In this paper, we report the complete nucleotide sequence of the $\mathrm{mt}$ genome of $S$. borealis strain F-4128. We describe the gene content, the genome organisation of the mitochondrial genome of $S$. borealis, and a comparative analysis of the known $\mathrm{mt}$ genomes of helotialean fungi. The main focus of this work is on genomic duplications and mobile genetic elements, such as introns and plasmid-related sequences. The distributions and potential origins of these elements are discussed.

\section{Materials and Methods}

\section{Sequencing, assembly, and annotation of the mitochondrial genome}

S. borealis F-4128 was obtained from the All-Russia Collection of Microorganisms (VKM). For DNA extraction, mycelia and sclerotia collected from the surface of agar were used. Total DNA was isolated by the SDS-CTAB method [29].

Mt genome sequencing was performed using a total genomic DNA sample without prior isolation of the mtDNA. The genome was sequenced with a Roche Genome Sequencer (GS) FLX, using the XL+ protocol for a shotgun genome library. The GS FLX run resulted in the generation of about $811 \mathrm{MB}$ of sequences with an average read length of 510 bp. The GS FLX reads were assembled into contigs using Newbler Assembler 2.8 (454 Life Sciences, Branford, CT). Two contigs, 190,990 bp (coverage 59X) and $10,928 \mathrm{bp}$ (coverage 62X), were identified as representing the mtDNA on the basis of extensive sequence similarity to known fungal mt genomes. The gaps between the contigs were closed by sequencing of corresponding PCR fragments.

The MFannot tool (http://megasun.bch.umontreal.ca/cgi-bin/ mfannot/mfannotInterface.pl) with default settings was used for mt genome annotation, which was adjusted manually by sequence alignment of deduced genes with their intron-less orthologs from related species. Putative proteins encoded by dubious ORFs were analysed by a BLAST homology search against the NCBI protein database. The codon frequency was determined using CodonW (http://www.molbiol.ox.ac.uk/cu/culong.html\#Codonw) for catenated ORFs for all protein-coding genes in the $S$. borealis $\mathrm{mt}$ genome.

Repeated sequences were identified by a BLASTN search of mt DNA against itself; matches with $\mathrm{E}$ - values $<\mathrm{e}^{-3}$ were taken into account.

The complete sequence of the mitochondrial genome of $S$. borealis F-4128 has been deposited in GenBank under the accession no. KJ434027.

\section{Genome comparison and phylogenetic analysis}

Whole mtDNA comparisons for the Peltigerales and Helotiales species Peltigera malacea, Peltigera membranacea, P. subalpina, B. fuckeliana, S. sclerotiorum, and S. borealis were performed using MAUVE 2.3.1 software [30]. The locally collinear blocks identified by MAUVE were compared with the annotated gene features.

For the phylogenetic analysis, we used 14 mitochondrial proteins, including subunits of the respiratory chain complexes (cox 1, cox 2, cox3, and cob), ATPase subunits (atp6, atp8, and atp9), and seven NADH dehydrogenase subunits (nad1, nad2, nad3, nad4, nad4L, nad5, and nad6). The list of 51 fungal species used to construct phylogenetic trees is shown in Table S3. Multiple sequence alignment was performed using the MUSCLE program of the MEGA5 [31] package, and poorly aligned positions and gap positions were removed with trimAl [32]. We used RAxML v7.6.6 to calculate the maximum likelihood phylogenetic tree with a gamma model of rate heterogeneity (four discrete rate categories and an estimated alpha parameter) and the WAG substitution matrix. We conducted 500 bootstrap replicates to define the support values on the tree. Also, we constructed a tree with a Bayesian method that produced a topology similar to the ML tree; bayesian analysis was performed using PhyloBayes with a JTT substitution model (4 discrete categories); trees were sampled using every 2 out of 638 generations; the first 150 trees were discarded as burn-in.

\section{Results and Discussion}

\section{General features of the $\mathrm{mt}$ genome of S. borealis}

The $\mathrm{mt}$ genome of $S$. borealis is a circular-mapping DNA molecule of 203,051 bp with a low GC content (32.1\%; Fig. 1). It contains a usual set of protein and RNA coding genes found in previously sequenced $\mathrm{mt}$ genomes of ascomycetes (Table 1). In addition to 31 tRNA genes and genes for the large and small ribosomal RNA ( $r n l$, $r n s)$, RNA-encoding genes include a predicted $r n p B$ gene encoding RNAse $\mathrm{P}$ (mtP-RNA), which is known to be responsible for tRNA processing [33].

Fourteen ORFs represent typical mt genes that encode subunits of the electron transport chain and of the ATP-synthase complex: seven subunits of electron transport complex I (nad1, nad2, nad3, nad4, nad4L, nad5, and nad6), one subunit of complex III (cob), three subunits of complex IV ( $\cos 1, \cos 2$, and $\cos 3)$, and three F0 subunits of the ATP-synthase complex (atp6, atp8, and atp9). In addition, there is a gene encoding the $40 \mathrm{~S}$ ribosomal protein S3 (rps3), located within an intron in the $r n l$, as is the case in most of filamentous ascomycetes [34]. All of these protein-coding ORFs are transcribed in the same direction and start with the canonical translation initiation codon, AUG. The preferred stop codon was UAA, with the exception of $\operatorname{cox} 3$, nad1, nad3, and nad6, which used UAG. ORFs encoding typical protein-coding mt genes contain 52 introns, the $r n l$ gene contains 8 introns, and one intron was found in rns.

In addition to typical mitochondrial genes, we also found 80 ORFs, including 61 located within introns, and 19 free-standing ORFs (Table S4). 52 intronic ORFs were predicted to encode proteins that exhibit similarities to homing endonucleases of LAGLIDADG (30 ORFs) and GIY-YIG (22 ORFs) families. 9 intronic ORFs were predicted to encode hypothetical proteins. Among free-standing ORFs, we found 2 putative homing endonuclease genes, three ORFs that are truncated fragments of DNA polymerase and RNA polymerase genes, and 14 ORFs encoding hypothetical proteins. At least 21 ORFs are probable pseudogenes, since their predicted protein products contain incomplete functional domains (Table S4).

Some fungal mitochondrial genomes contain a high fraction of repeat sequences which could account for an increase in the genome size, as observed for the mt genomes of Agaricus bisporus [35] and R. solani [7]. Dot plot analysis shows the lack of long duplicated regions in the $S$. borealis $\mathrm{mt}$ genome (Figure S1), and a BLASTN similarity search reveals that repeated sequences account for only about $6.6 \%$ of $S$. borealis mtDNA. Similar values were obtained for the mt genomes of $S$. sclerotiorum $(6.5 \%)$, B. fuckeliana $(4.2 \%)$ and $P$. subalpina $(6.0 \%)$, suggesting that the accumulation of repeated sequences is not the primary reason for mitochondrial genome expansion in $S$. borealis. 


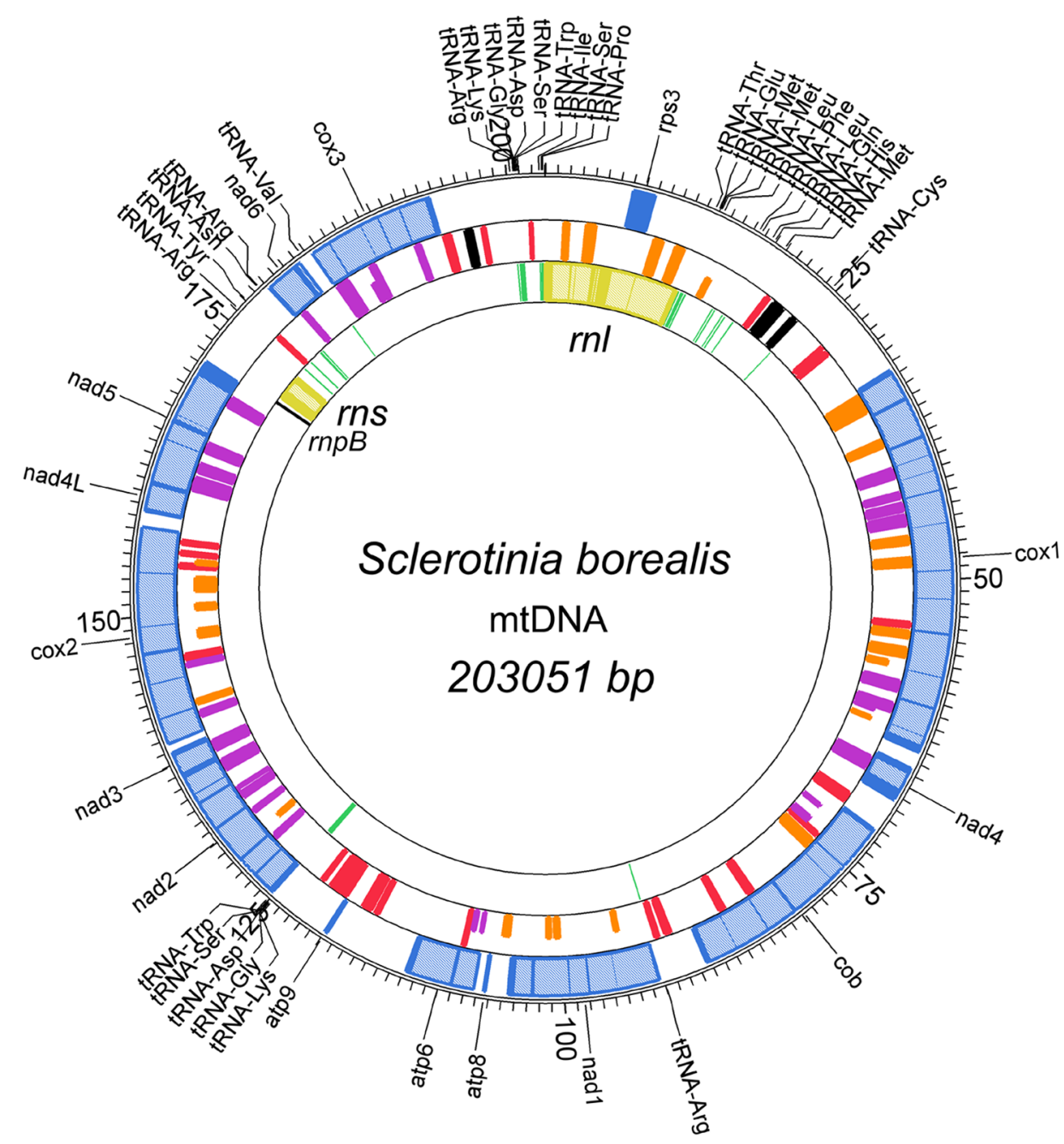

Figure 1. Map of the mitochondrial genome of $S$. borealis. The first ring from the outside represents the $S$. borealis core mitochondrial proteincoding genes and rps3 (blue boxes). The second ring from the outside represents the hypothetical ORFs in red, ORFs for proteins containing GIY-YIG domains in orange, ORFs for proteins containing LAGLIDADG domains in purple, and fragments of DNA polymerase B and RNA polymerase genes in black. Full-size boxes indicate proteins containing complete GIY-YIG or LAGLIDADG domains, and half-size boxes indicate incomplete domains. The third ring represents the $r n s$ and $r n l$ genes in yellow, $r n p B$ in black and tRNA genes in green. Exons are indicated by dark colours, and introns are in light colours. All genes are oriented clockwise except for one fragment of DNA polymerase B (22818-21772 nt).

doi:10.1371/journal.pone.0107536.g001

\section{Transfer RNAs and genetic code}

Similar to many mt genomes of Pezizomycotina, tRNA genes are clustered in the mtDNA of $S$. borealis (Fig. 1). The main cluster, consisting of 20 tRNAs, is located around the $r n l$ gene. Two clusters including five and four tRNA genes are located between atp 9 and nad2, and between $r n s$ and nad6, respectively. A set of 31 tRNA genes (Table S1) is sufficient to decode all of the codons present in the predicted ORFs, except for GCN, since we did not find the corresponding tRNA gene. The presence of tRNA-W (anticodon UCA) recognising the TGA codon suggests that $S$. borealis $\mathrm{mt}$ protein coding genes are translated according to genetic code 4 (yeast mitochondrial genetic code).

The frequency of codon usage, summarised in Table S2, shows that all possible codons are used. AT-rich codons are much more abundant, reflecting the high AT content of the S. borealis $\mathrm{mt}$ genome. Codons for amino acids with nonpolar side chains (Phe, Leu, and Ile) are very frequent, which is not surprising given the hydrophobic nature of the proteins of respiratory membrane complexes. It is likely that abundant AUA isoleucine codons are read by one of the three predicted tRNA-M after the cytosine to lysidine modification of the CAU anticodon, such as that occurs in some plant and fungal mitochondrial genomes [36].

\section{Phylogenetic analysis}

Previously, phylogenetic relationships among Pezizomycotina, a subphylum of Ascomycota comprising the order Helotiales, were established based on the comparison of five nuclear genes $(S S U$ $r D N A, L S U$ rDNA, RPB1, RPB2, and EF-1 $\alpha$ ) [37]. Here, we used 14 core $\mathrm{mt}$ proteins for the phylogenetic analysis of Ascomycota. Our phylogenetic analysis, performed by both Bayesian (Fig. 2) and maximum likelihood (Fig. S2) methods, produced similar trees and confirmed the affiliation of $S$. borealis with the order of Helotiales, together with $P$. subalpina, $G$. lozoyensis, M. brunnea, Rhynchosporium sp., B. fuckeliana, and $S$. sclerotiorum. P. subalpina, G. lozoyensis, $M$. brunnea and Rhynchosporium sp. appeared to form a separate branch among the analysed Helotiales, while Sclerotinia and Botrytinia species formed another branch and probably represent a single genus, 
Table 1. Genes encoding 14 typical mitochondrial proteins and the ribosomal RNA subunits in S. borealis mitochondrial genome.

\begin{tabular}{lllll}
\hline & & & & \\
\hline Gene & Introns & Gene size, bp & CD size, bp & \% of intronic sequences \\
\hline cox1 & 13 & 31646 & 2073 & 93.4 \\
cox2 & 6 & 17584 & 756 & 95.7 \\
cox3 & 5 & 10309 & 810 & 92.1 \\
cob & 6 & 17133 & 1197 & 93.0 \\
nad1 & 5 & 12150 & 1092 & 91.0 \\
nad2 & 6 & 11796 & 1698 & 85.6 \\
nad3 & 1 & 1891 & 441 & 76.7 \\
nad4 & 1 & 2856 & 1470 & 48.5 \\
nad4L & 1 & 2221 & 270 & 87.8 \\
nad5 & 4 & 10691 & 1980 & 81.5 \\
nad6 & 2 & 3137 & 693 & 77.9 \\
atp6 & 2 & 5566 & 771 & 86.1 \\
atp8 & 0 & 147 & 147 & 0 \\
atp9 & 0 & 225 & 225 & 0 \\
rnl & 8 & 13441 & 2842 & 78.9 \\
rns & 1 & 2598 & 1532 & 41.0 \\
\hline doi:10.1371/journal.pone.0107536.t001 & & \\
\hline
\end{tabular}

since $S$. borealis is a sister lineage of a group comprising $B$. fuckeliana and S. sclerotiorum. (Fig. 2). Five classes of filamentous ascomycetes are clearly distinguished as monophylectic groups (Fig. 2 and S2): Leotiomycetes (represented by Helotiales), Lecanoromycetes (represented by Peltigerales), Sordariomycetes, Dothideomycetes and Eurotiomycetes. Most nodes in these trees have high bootstrap values, which indicate the robustness of the computed trees. In previous studies, Sordariomycetes and Leotiomycetes were resolved with moderate support, as they share a most recent common ancestor, with Lecanoromycetes as a sister group [37], [38]. The present analysis identifies Peltigerales as the closest relative of the Leotiomycetes, while Sordariomycetes is a more deeply branching lineage (Fig. 2). Phylogenetic analysis involving more representatives of Lecanoromycetes and Leotiomycetes would help to clarify relationships between these lineages.

\section{Gene order comparison}

Previously, the mt genomes of three helotialean species, $P$. subalpina, S. sclerotiorum and B. fuckeliana, were compared by Duo et al., 2012 [16]. We extended this analysis and included the data on the order of conserved genes in the mtDNAs of $S$. borealis, G. lozoyensis, M. brunnea, Rhynchosporium sp., and two Peltigera species. Genome alignments (Fig. 3) reveal rearrangements between Peltigerales and Helotiales species, as well as within Helotiales, between the P. subalpina / M. brunnea / Rhynchosporium sp. group and the Sclerotinia/Botryotinia group. The arrangement of $\mathrm{mt}$ genes in $G$. lozoyensis deviates significantly from both groups. In contrast, the three compared species of Sclerotiniaceae have almost complete synteny in gene order, with minor differences (location of atp9) attributed to duplication events. The gene order in the mt genomes of the second group of Helotiales, comprising P. subalpina, M. brunnea and Rhynchosporium sp., is also well conserved and differs only in the presence/ absence of atp9 and location of $\operatorname{rps} 3$.

The conservation of gene order among Sclerotinia/Botryotinia species contrasts drastically with the differences in mtDNA size. $S$. borealis has the largest genome (203.1 kbp), while other species have smaller mtDNAs: $128.9 \mathrm{kbp}$ in S. sclerotiorum and $82.2 \mathrm{kbp}$ in B. fuckeliana.

\section{S. borealis mtDNA contains duplicated copies of some genes}

The large size of $S$. borealis mtDNA may be caused by duplications of some genome regions. Such events that resulted in the appearance of truncated extra copies of atp 6 and atp 9 genes were identified. The mtDNA of $S$. borealis contains two ORFs with homology to atp9 (Fig. 4). The first ORF is located upstream of nad2 and probably encodes an intact 74-aa long Atp9 protein. The second atp9-like ORF 0044 is situated between the $c o b$ and nad 1 genes. It consists of a full-size atp 9 sequence $(96 \%$ identity over 222-bp coding sequence) lacking a stop codon, and followed by an unrelated sequence capable of encoding the 213-aa Cterminal peptide.

Analysis of the $c o b-n a d 2$ region of $S$. borealis and B. fuckeliana revealed extensive synteny between these regions (Fig. 4). However, in B. fuckeliana, an intact copy of atp9 is located between cob and nad1, while the second copy between atp6 and nad2 is missing. Two copies of the atp9-related ORFs were also found in related species, including $P$. subalpina [16], thus indicating that gene duplication and the subsequent truncation of one of the two copies of atp9 is a common feature of $\mathrm{mt}$ genomes of helotialean species.

In another case, duplication of relatively short piece of gene was predicted. The region between nad1 and nad2 also contains two ORFs showing sequence similarity to atp6. The first is an intact full-size atp6, while the second downstream 384-bp long ORF 0116 contains an internal 183-bp fragment of atp6 (80\% nucleotide sequence identity). Such an arrangement may have resulted from a tandem duplication event. An additional truncated copy of the atp6 gene is also present in B. fuckeliana mtDNA (Fig. 4). 


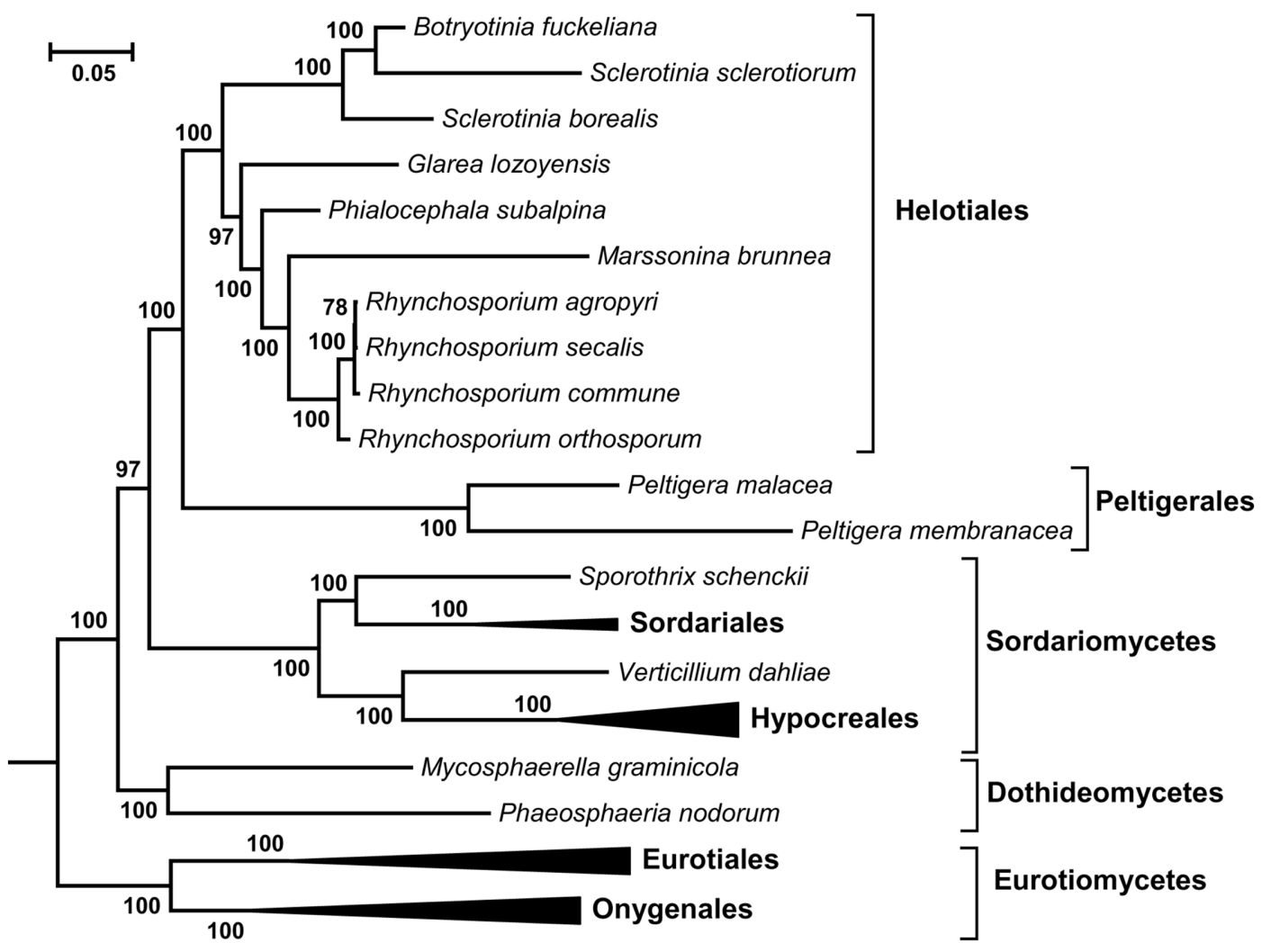

Figure 2. The phylogenetic tree was calculated from the multiple sequence alignment of concatenated mtDNA-encoded proteins of 51 fungal species. A dataset of 14 proteins was used, and topology was inferred using Bayesian method. Numbers above the nodes indicate bootstrap support values. The tree is drawn to scale, with branch lengths measured by the number of substitutions per site. Species analysed are shown in Table S3; only the Ascomycota branch of the whole tree is shown. doi:10.1371/journal.pone.0107536.g002

Introns in S. borealis mtDNA

Sequences encoding 14 typical mitochondrial proteins and the ribosomal RNA subunits $(r n l$ and $r n s)$ totalled $18 \mathrm{~kb}$ and represent only $9 \%$ of the entire genome. The 61 introns (Table 1) identified in core mitochondrial protein-coding genes and the $\mathrm{rns}$ and $\mathrm{rnl}$ genes account for $125 \mathrm{~kb}$ ( $62 \%$ of the genome). The introns range

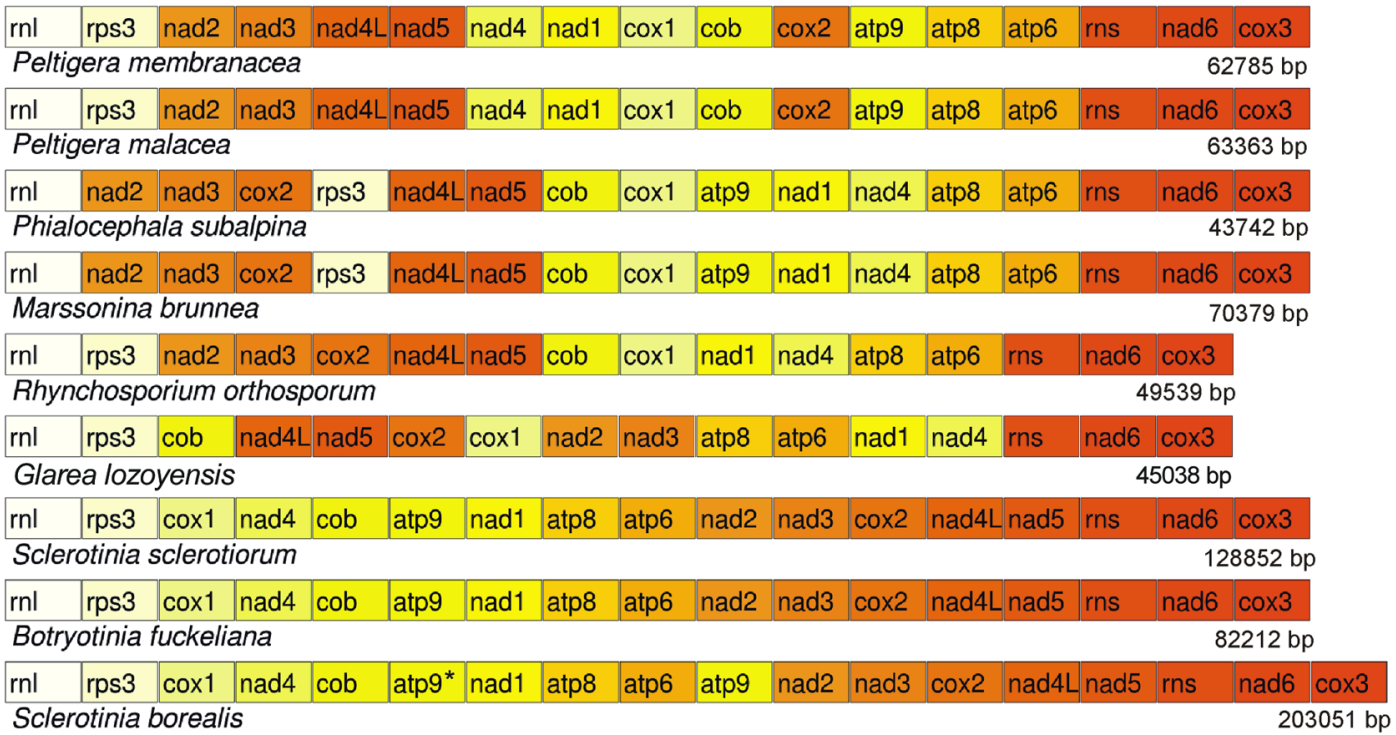

Figure 3. Mitochondrial genome rearrangements observed among Helotiales and Peltigerales. The second truncated copy of atp9 in S. borealis mtDNA is marked by an asterisk. doi:10.1371/journal.pone.0107536.g003 


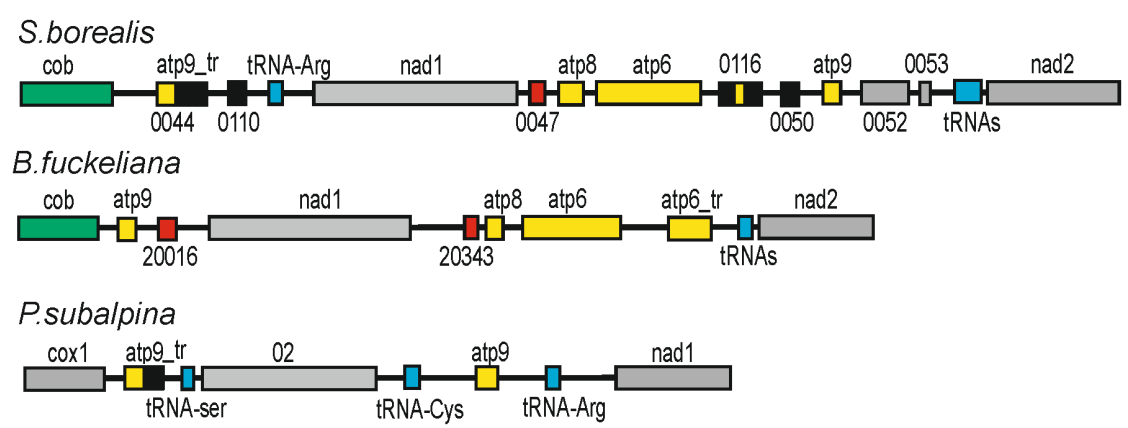

Figure 4. Genome organisation of the region around atp9genes for the helotialean species. Boxes represent ORFs and tRNA genes (blue, tRNA genes; yellow, atp ORFs and gene fragments; green, cob ORFs and gene fragments; black, inserts in truncated copies of genes; red, intronic HEGlike ORFs; grey, other ORFs). The second truncated copies of atp6 and atp9 are shown as atp6_tr and atp9_tr, respectively. doi:10.1371/journal.pone.0107536.g004

in size from $251 \mathrm{nt}$ for intron 1 in the $r n l$ gene, to $4300 \mathrm{nt}$ for intron 5 in the $\operatorname{cox} 2$ gene. These introns are scattered throughout the genome (Fig. 1 and Table 1) and are found in 12 of the 15 protein-coding genes (52 introns), in the $r n l$ gene (8 introns) and in the rns (1 intron). Specifically, 13 introns were found in the cox 1 gene, accounting for $93.4 \%$ of the gene sequence. The $c o b$, nad 2 , and $\operatorname{cox} 2$ genes have six introns each. The nad1, cox3, and nad5 genes possess four introns each. The nad6 and atp6 genes have two introns each, while the $\operatorname{nad} 4, \operatorname{nad} 4 L$, and nad3 contain only a single intron each.

$35 \mathrm{ORF}_{\mathrm{s}}$ related to homing endonucleases (HEG) [39] of the LAGLIDADG (23 ORFs) and GIY-YIG families (12 ORFs) were found in introns. In addition, 17 HEG-related intronic HEGrelated ORFs are probably pseudogenes, since their predicted products contain incomplete LAGLIDADG (7 ORFs) or GIYYIG (10 ORFs) domains (Table S4). Two HEG-related ORFs containing a partial GIY-YIG domain were identified outside of core mitochondrial genes (Table S4). These free-standing HEGrelated ORFs have limited similarity $(35-53 \%$ amino acid sequence identity) to intronic ORFs of phylogenetically remote fungal species, but no significant similarity to any $S$. borealis proteins (Table S4), which suggests their acquisition via lateral gene transfer rather than by intragenomic proliferation.

We compared the locations of introns in the mt genomes of $S$. borealis and the phylogenetically related organisms $B$. fuckeliana, Rhynchosporium commune, Rhynchosporium orthosporum, $P$. malacea, and P. membranacea (Table 2). S. sclerotiorum was not included in this analysis since its mitochondrial genome was not annotated and intron locations are unknown. Comparative analysis showed that most introns are present in two genes: cox 1 and $c o b$ (Table 2). Many orthologous introns are located in the same positions in all of the analysed species.

The $\operatorname{cox} 1$ gene is the most common target for insertions of group I introns. Introns in this gene were found in different taxonomic groups, and the position of insertion may be used for analysis of intron mobility during evolution [26]. Among the 13 introns found in the cox 1 gene in $S$. borealis, eight introns $(1,2,3,4,6,8,9,10)$ have a high sequence identity to orthologous introns found in the same positions in cox 1 genes of other Helotiales and Peltigerales (Table S5), suggesting a common origin. For example, the nucleotide sequence of intron 3 of the $S$. borealis cox 1 is $95 \%$ identical to the corresponding cox 1 intron in B. fuckeliana (Table S5). Three introns $(5,11,13)$ have a higher sequence similarity to introns of other fungi (Table S5), suggesting their acquisition through horizontal transfer.

Overall, only 18 introns found in protein-coding genes showed the highest nucleotide sequences similarity to $\mathrm{mt}$ introns of
Helotiales (17 introns) and Peltigerales (1 introns), while 29 introns are most similar to introns of other fungal lineages, in some instances even distantly related (e.g., intron 6 of nad2 is homologous to the mtDNA intron of the oomycete Albugo laibachii). Two cox 1 introns (7 and 12) and three introns located in other protein-coding genes ( $c o b$ intron 4, nad1 intron 4 and nad6 intron 2) show no homology to other fungal introns (Table S5), and also may originate from phylogenetically remote fungal species.

Among 9 introns found in RNA-coding genes, only three are most similar to rRNA introns of Helotiales, while four share the highest identity with introns of mtDNA of other fungi, and two introns did not show significant similarity to sequences deposited in GenBank.

Analysis of nucleotide sequences of the orthologous introns in the $\operatorname{cox} 1$ gene reveal events of multiple insertions during intron evolution (Fig. 5A). The first 2061 bp long intron has two regions with $76-80 \%$ nucleotide sequence identity with the $1308 \mathrm{bp}$ intron present at the same position in the $\operatorname{cox} 1$ genes of $P$. malacea [40]. The central region (about bp 500-1250) of this intron in $S$. borealis is similar to an internal region of a $2499 \mathrm{bp}$ long intron of the cob gene of phylogenetically distant fungus, Podospora anserina of the order Sordariales, suggesting the additional insertion of a new sequence into an already present intron. This insertion occurred in-frame within the HEG-like ORF present in the first intron, thus resulting in the appearance of a second GIYYIG catalytic domain in the intron-encoded protein (Fig 5A). The closest relative of $S$. borealis, the helotialean fungus $B$. fuckeliana, harbours in this position an intron with a dissimilar nucleotide sequence. It can be assumed that insertion of the intron occurred in a common ancestor of Peltigerales and Helotiales, and it was then replaced by another intron in B. fuckeliana, while an extra insertion occurred in $S$. borealis (Fig5A).

Three insertions disrupting the HEG-like ORF occur in intron 8 of cox 1 in S. borealis (Fig 5B). Excluding these insertions, the nucleotide sequence of this intron is about $80 \%$ identical to the corresponding introns present at the same positions in $P$. malacea. An orthologous but shorter intron is present at this position in $B$. fuckeliana; it may result from internal deletion within the parental intron (Fig 5B).

Overall, our analysis reveals possible evolutionary events, including insertions of introns and the horizontal transfer of introns from remote fungal species, that have led to an increase in the size of the $S$. borealis $\mathrm{mt}$ genome. 


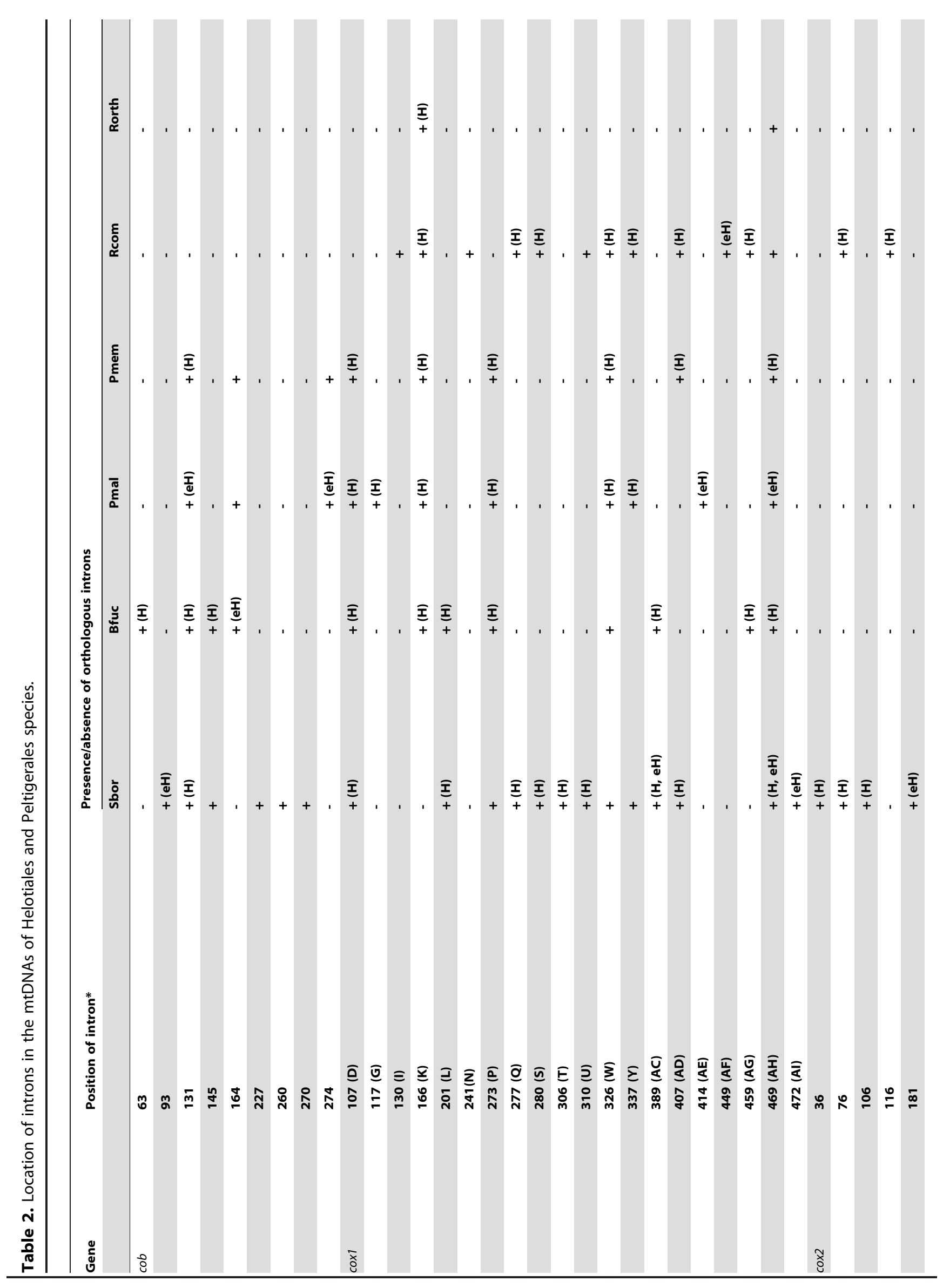




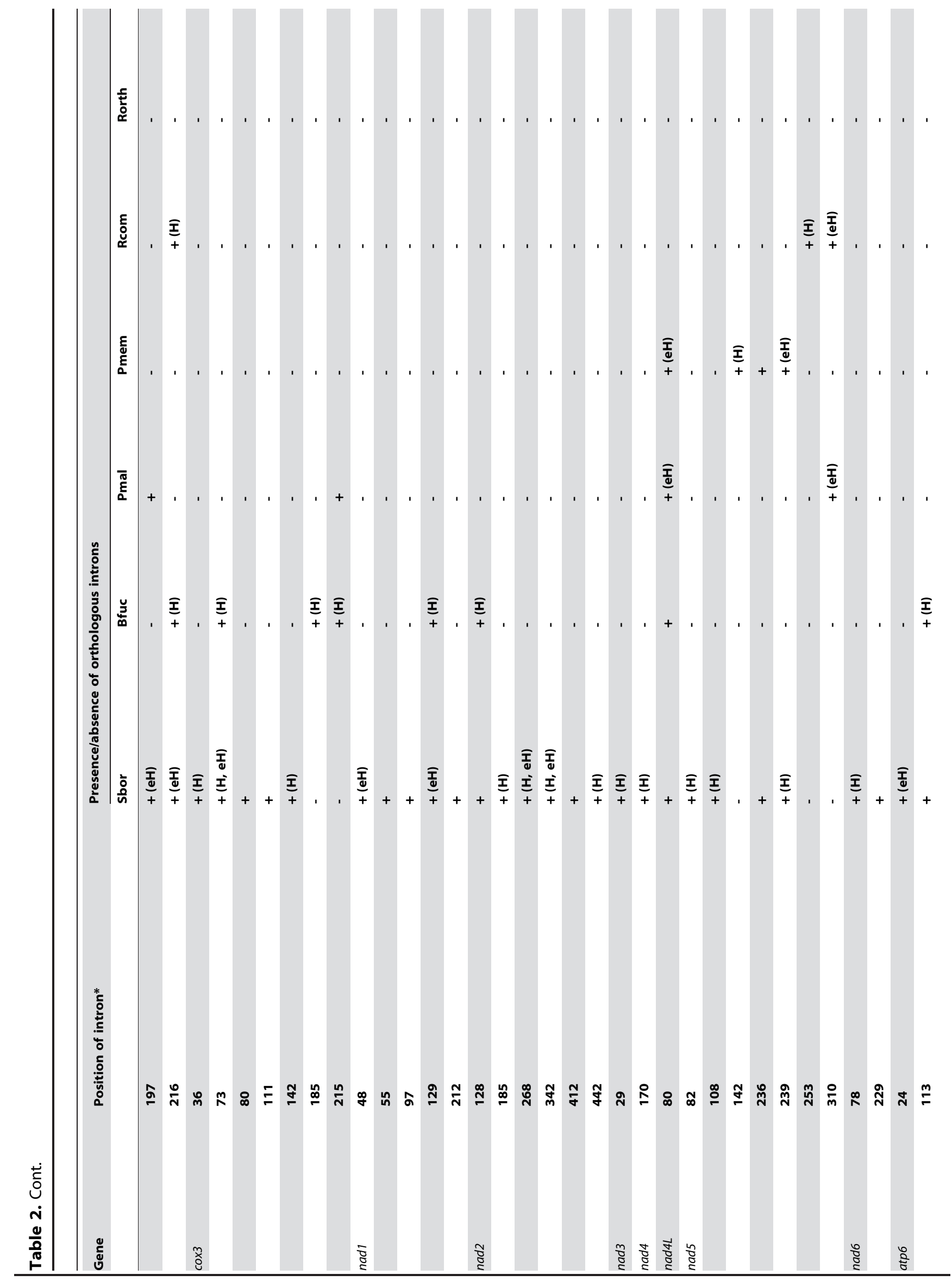




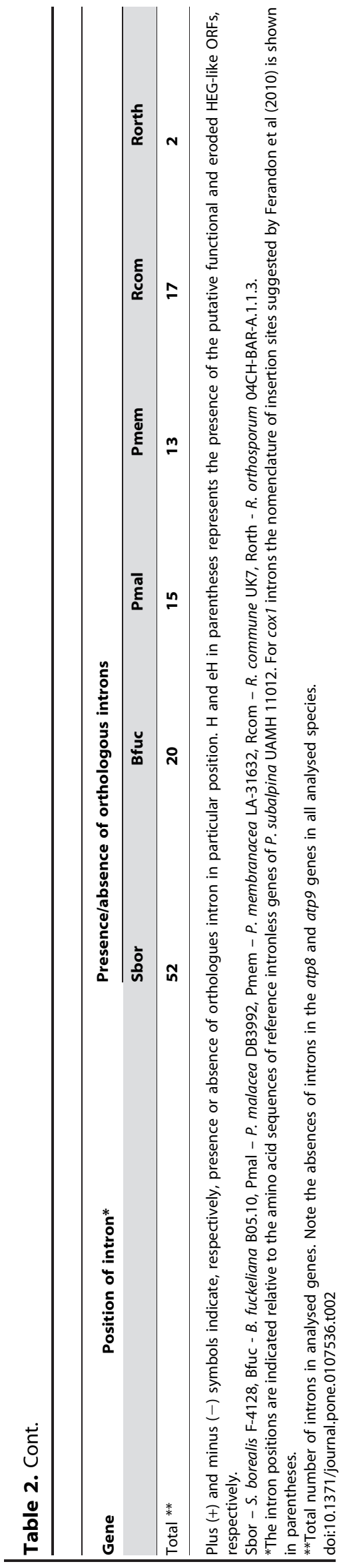

\section{Plasmid-like sequences integrated in the mtDNA}

In addition to the introns, plasmids represent other types of mobile genetic elements often found in the mitochondria of fungi and plants [41]. These plasmids can be linear or integrated into the mt genome [42]. Most contain two ORFs, one encoding a family B DNA polymerase, and the second encoding a DNAdependent RNA polymerase subunit, as well as several other ORFs [42]. The majority of $\mathrm{mt}$ plasmids have been predicted based on the analysis of genomic data [43], [44]. It has been shown that such plasmids can be integrated into the mt genomes [43], [45], [46], [47], [48], [49]. Upon integration, they accumulate mutations and deletions, and are finally eliminated during evolution. A region in the $S$. borealis $\mathrm{mtDNA}$ located between the $r n l$ and $\operatorname{cox} 1$ genes contains an ORF 0018 encoding a $1047 \mathrm{bp}$ fragment of DNA polymerase B, and a nearby located 540 bp fragment of the RNA polymerase gene (ORF 0019). The second region, located downstream of the $\operatorname{cox} 3$ gene, contains a 591 bp long ORF 0093 for the DNA polymerase B fragment. It can be assumed that these regions may have resulted from an ancient integration of plasmids, which were then mostly eliminated from the mtDNA of $S$. borealis. The sequences of the two DNA polymerase $\mathrm{B}$ genes have a low homology $(22 \%$ amino acid identity), suggesting the different origin of these two insertions.

\section{Conclusions}

Our data on the organisation of the mt genome of $S$. borealis, and its comparison with mtDNA sequences of related species, reveal its specific features and the general characteristics of mtDNAs of the order Helotiales [16,40]. Our comparative analysis indicates that the 203,051 bp long $S$. borealis $\mathrm{mt}$ genome is the second largest fungal $\mathrm{mt}$ genome sequenced to date, after the 235,849 bp long mtDNA of the basidiomycete $R$. solani RhslAP [7]. The next largest mitochondrial genomes are the $R$. solani AG1-IB (162,751 bp) [50] and A. bisporus (135,005 bp) [35]. Expansion of the mitochondrial genome size in $R$. solani $\mathrm{Rhs} 1 \mathrm{AP}$ was driven by accumulation of introns, HEG-like genes and hypothetical genes, but the most peculiar feature was the presence of various interspersed repeats, which occupy about one third of the genome [7].

In contrast, the major contributors to the large size of the $S$. borealis $\mathrm{mt}$ genome are introns, while different repeats accounted for less than $7 \%$ of the genome sequence. The number of introns found in $S$. borealis (61) is higher than in the mt genomes of $R$. solani Rhs1AP (43) and $A$. bisporus (46). Introns identified in the core mitochondrial genes accounted for $125 \mathrm{~kb}$. The actual contribution of intron-related sequences to the genome size is probably higher, since their insertion in intergenic regions, evidenced by the finding of several free-standing HEG-related ORFs, will increase their proportion in the mtDNA. The mt genome of $S$. borealis contains a large number of introns found in different lineages of fungi, including phylogenetically distant ones, thus indicating the important role of the horizontal transfer of introns in the evolution of $\mathrm{mt}$ genomes. Extension of intergenic regions, accounting for about $50 \mathrm{~kb}$ ( $23 \%$ of the genome), is another major contributor to the large size of the $S$. borealis $\mathrm{mt}$ genome. Duplications of some regions and insertion of plasmids also increase the size of the genome.

Interestingly, phylogenetically related fungi of the orders Peltigetales and Helotiales differ in mtDNA length and the number of introns. A notable feature is the complete absence of introns in the $\mathrm{mt}$ genome of $P$. subalpina [16]. To the contrary, many introns found in $S$. borealis are also present in the mtDNAs of the representatives from the more deeply branching order Peltigerales (P. malaceae and P. membranaceae) (Table S5). These 
(A)

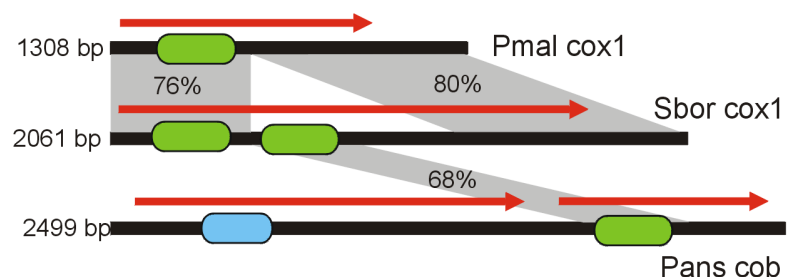

(B)

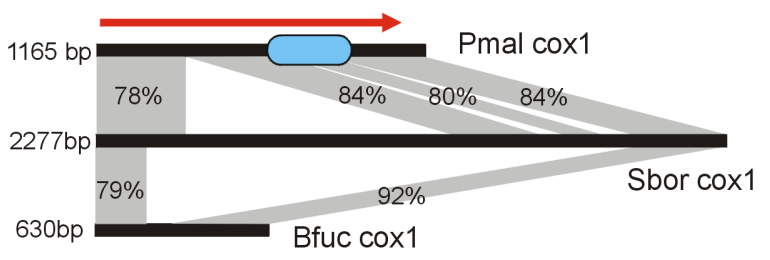

Figure 5. Structure of the $S$. borealis cox 1 gene introns 1 (A) and 8 (B). Introns are represented by black horizontal bars; the sizes are drawn to scale. Red arrows show HEG-like ORFs. Green and blue rectangles show, respectively, catalytic GIY-YIG_bl1_like (cd10445) and LAGLIDADG_1 (pfam00961) domains. Nucleotide sequence similarity is indicated by grey areas between introns. Sbor $-S$. borealis, Bfuc $-B$. fuckeliana, Pmal - P. malacea, Pans - P. anserina.

doi:10.1371/journal.pone.0107536.g005

observations imply a dynamic pattern of intron acquisition and loss during evolution: in some lineages, introns were completely lost, while in others, extensive accumulation of introns occurred. It is likely that the $S$. borealis genome has efficient mechanisms for intron acquisition and retention, but the nature of these mechanisms remains unknown.

\section{Supporting Information}

Figure $S 1$ Dot plot analysis of $S$. borealis mtDNA performed with Dotmatcher. (http://emboss.bioinformatics. nl/cgi-bin/emboss/dotmatcher). The main diagonal represents the sequence's alignment with itself; lines off the main diagonal

\section{References}

1. Hoshino T, Terami F, Tkachenko OB, Tojo M, Matsumoto N (2010) Mycelial growth of the snow mold fungus, Sclerotinia borealis, improved at low water potentials: an adaptive to frozen environment. Mycosciences 51: 98-103.

2. Tomiyama K (1955) Studies of the snow blight disease of winter cereals. Hokkaido Natiolnal Agricultural Experimantal Station Rep 47: 1-234.

3. Pantou MP, Kouvelis VN, Typas MA (2006) The complete mitochondrial genome of the vascular wilt fungus Verticillium dahliae: a novel gene order for Verticillium and a diagnostic tool for species identification. Curr Genet 50: 125136.

4. Kouvelis VN, Sialakouma A, Typas MA (2008) Mitochondrial gene sequences alone or combined with ITS region sequences provide firm molecular criteria for the classification of Lecanicillium species. Mycol Res 112: 829-844.

5. Ballard JW, Whitlock MC (2004) The incomplete natural history of mitochondria. Mol Ecol 13: 729-744.

6. Basse CW (2010) Mitochondrial inheritance in fungi. Curr Opin Microbiol 13: $712-719$.

7. Losada L1, Pakala SB, Fedorova ND, Joardar V, Shabalina SA, et al. (2014) Mobile elements and mitochondrial genome expansion in the soil fungus and potato pathogen Rhizoctonia solani AG-3. FEMS Microbiol Lett. 352(2): 165173.

8. Gray M, Burger G, Lang B (1999) Mitochondrial evolution. Science 283(5407): 1476-1481.

9. Lavin JL, Oguiza JA, Ramirez L, Pisabarro AG (2008) Comparative genomics of the oxidative phosphorylation system in fungi. Fungal Genet Biol 45 (9): 12481256.

10. Wu Y, Yang J, Yang F, Liu T, Leng W, et al. (2009) Recent dermatophyte divergence revealed by comparative and phylogenetic analysis of mitochondrial genomes. BMC Genomics 10: 238. represent repetitive patterns within the sequence. Each dot represents a 100 bp significantly matching segment.

(PDF)

Figure S2 The phylogenetic tree was calculated from the multiple sequence alignment of 14 concatenated mtDNA-encoded proteins. Topology was inferred using Maximum-Likelhood method. Numbers above the nodes indicate bootstrap support values. The tree is drawn to scale, with branch lengths measured by the number of substitutions per site. Species analyzed are shown in the Table S3, only Ascomycota branch of the whole tree is shown.

(PDF)

Table S1 The codon-anticodon recognition pattern and tRNA genes identified in $S$. borealis mitochondrial genome.

(DOC)

Table S2 Codon usage of protein-coding genes in $S$. borealis mitochondrial genome.

(DOC)

Table S3 List of mt genomes used for phylogenetic studies.

(DOC)

Table S4 List of ORFs identified in $S$. borealis mitochondrial genome.

(XLS)

Table S5 Sequence similarity betwen mt introns of $S$. borealis and introns of other organisms.

(XLS)

\section{Acknowledgments}

We thank the anonymous reviewers for their careful reading of our manuscript and their insightful comments and suggestions.

\section{Author Contributions}

Conceived and designed the experiments: AVM NVR. Performed the experiments: AVM VVK. Analyzed the data: AVM AVB. Contributed reagents/materials/analysis tools: ANI. Wrote the paper: AVM NVR.

11. Sethuraman J, Majer A, Friedrich NG, Edgell DR, Hausner G (2009) Genes within genes: multiple LAGLIDADG homing endonucleases target the ribosomal protein S3 gene encoded within an $\mathrm{rnl}$ Group I intron of Ophiostoma and related taxa. Mol Biol Evol 26(10): 2299-2315.

12. Monteiro-Vitorello CB, Hausner G, Searles DB, Gibb EA, Fulbright DW, et al. (2009) The Cryphonectria parasitica mitochondrial rns gene: Plasmid-like elements, introns and homing endonucleases. Fungal Genet Biol 46(11): 837848.

13. Gibb E, Hausner G (2005) Optional mitochondrial introns and evidence for a homing-endonuclease gene in the mtDNA rnl gene in Ophiostoma ulmi s. lat. Mycol Res 109: 1112-1126.

14. O'Brien EA, Zhang Y, Wang E, Marie V, Badejoko W, et al. (2009) GOBASE: An organelle genome database. Nucleic Acids Res 37: 946-950.

15. Korab-Laskowska M, Rioux P, Brossard N, Littlejohn TG, Gray MW, et al. (1998) The Organelle Genome Database Project (GOBASE). Nucleic Acids Res 26: $138-144$.

16. Duò A, Bruggmann R, Zoller S, Bernt M, Grunig CR (2012) Mitochondrial genome evolution in species belonging to the Phialocephala fortinii s.l. Acephala applanata species complex. BMC Genomics 13: 166.

17. Kouvelis VN, Ghikas DV, Typas MA (2004) The analysis of the complete mitochondrial genome of Lecanicillium muscarium (synonym Verticillium lecanii) suggests a minimum common gene organization in mtDNAs of Sordariomycetes: phylogenetic implications. Fungal Genet Biol 41: 930-940.

18. Cardoso MA, Tambor JH, Nobrega FG (2007) The mitochondrial genome from the thermal dimorphic fungus Paracoccidioides brasiliensis. Yeast 24: 607-616.

19. Cummings DJ, McNally KL, Domenico JM, Matsuura ET (1990) The complete DNA sequence of the mitochondrial genome of Podospora anserina. Curr Genet 17: $375-402$. 
20. van de Sande WWJ (2012) Phylogenetic analysis of the complete mitochondrial genome of Madurella mycetomatis confirms its taxonomic position within the order Sordariales. PLoS One 7(6): e38654.

21. Youssar L, Grüning BA, Günther S, Hüttel W (2013) Characterization and phylogenetic analysis of the mitochondrial genome of Glarea lozoyensis indicates high diversity within the order Helotiales. PLoS One 8(9): e74792.

22. Torriani SF, Penselin D, Knogge W, Felder M, Taudien S, et al. (2014) Comparative analysis of mitochondrial genomes from closely related Rhynchosporium species reveals extensive intron invasion. Fungal Genet Biol 62: $34-42$.

23. Bullerwell CE, Gray MW (2004) Evolution of the mitochondrial genome: protist connections to animals, fungi and plants. Curr Opin Microbiol 7: 528-534.

24. Bullerwell CE, Forget L, Lang BF (2003) Evolution of monoblepharidalean fungi based on complete mitochondrial genome sequences. Nucleic Acids Res 31: 1614-1623.

25. Burger G, Gray M, Lang B (2003) Mitochondrial genomes: anything goes. Trends Genet 19(12): 709-716.

26. Ferandon C, Moukha S, Callac P, Benedetto J-P, Castroviejo M, et al. (2010) The Agaricus bisporus coxl gene: the longest mitochondrial gene and the largest reservoir of mitochondrial group I introns. PLoS One 5: e14048.

27. Goddard MR, Burt A (1999) Recurrent invasion and extinction of a selfish gene. Proc Natl Acad Sci USA. 96(24): 13880-13885.

28. Cusimano N, Zhang LB, Renner SS (2008) Reevaluation of the cox 1 group I intron in Araceae and angiosperms indicates a history dominated by loss rather than horizontal transfer. Mol Biol Evol 25(2): 265-276.

29. Milligan BG (1998) Total DNA isolation. In: Hoelzel AR, editor. Oxford, Molecular Genetic Analysis of Population: A Practical Approach, 2nd Edition pp. 29-64.

30. Darling ACE, Mau B, Blattner FR, Perna NT (2004) Mauve: Multiple Alignment of Conserved Genomic Sequence With Rearrangements. Genome Res 14(7): 1394-1403.

31. Tamura K, Peterson D, Peterson N, Stecher G, Nei M, et al. (2011) MEGA5: molecular evolutionary genetics analysis using maximum likelihood, evolutionary distance, and maximum parsimony methods. Mol Biol Evol 28(10): 27312739 .

32. Capella-Gutiérrez S, Silla-Martínez JM, Gabaldón (2009) trimAl: a tool for automated alignment trimming in large-scale phylogenetic analyses. Bioinformatics 25(15): 1972-1973.

33. Seif ER, Forget L, Martin NC, Lang BF (2003) Mitochondrial Rnase P RNAs in ascomycete fungi: lineage-specific variations in RNA secondary structure. RNA 9: $1073-1083$

34. Sethuraman J, Majer A, Iranpour M, Hausner G (2009) Molecular evolution of the mtDNA encoded $r p s 3$ gene among filamentous ascomycetes fungi with an emphasis on the Ophiostomatoid fungi. J Mol Evol 69(4): 372-385.

35. Ferandon C, Xu J, Barroso G (2013) The $135 \mathrm{kbp}$ mitochondrial genome of Agaricus bisporus is the largest known eukaryotic reservoir of group I introns and plasmid-related sequences. Fungal Genet Biol 55: 85-91.
36. Weber F, Dietrich A, Weil JH, Maréchal-Drouard L (1990) A potato mitochondrial isoleucine tRNA is coded for by a mitochondrial gene possessing a methionine anticodon. Nucleic Acids Res 18: 5027-5030.

37. Spatafora JW, Sung GH, Johnson D, Hesse C, O'Rourke B, et al. (2006) A fivegene phylogeny of Pezizomycotina. Mycologia 98: 1018-1028.

38. Lumbsch HT, Schmitt I, Lindemuth R, Miller A, Mangold A, et al. (2005) Performance of four ribosomal DNA regions to infer higher-level phylogenetic relationships of inoperculate euascomycetes (Leotiomyceta). Mol Phylogen Evol 34: 512-524.

39. Stoddard BL (2011). Homing endonucleases: from microbial genetic invaders to reagents for targeted DNA modification. Structure 19(1): 7-15.

40. Xavier BB, Miao VP, Jónsson ZO, Andrésson ÓS (2012) Mitochondrial genomes from the lichenized fungi Peltigera membranacea and Peltigera malacea: features and phylogeny. Fungal Biol 16(7): 802-814.

41. Cahan P, Kennell JC (2005) Identification and distribution of sequences having similarity to mitochondrial plasmids in mitochondrial genomes of filamentous fungi. Mol Genet Genom 273: 462-473.

42. Griffiths AJ (1995). Natural plasmids of filamentous fungi. Microbiol. Rev. 59(4): 673-685.

43. Mouhamadou B, Barroso G, Labarere J (2004). Molecular evolution of a mitochondrial polB gene, encoding a family B DNA polymerase, towards the elimination from Agrocybe mitochondrial genomes. Mol Genet Genom 272(3): 257-263.

44. Formighieri EF, Tiburcio RA, Armas ED, Medrano FJ, Shimo H, et al. (2008) The mitochondrial genome of the phytopathogenic basidiomycete Moniliophthora perniciosa is $109 \mathrm{~kb}$ in size and contains a stable integrated plasmid. Mycol Res 112: 1136-1152.

45. Robison MM, Horgen PA (1996). Plasmid RNA polymerase-like mitochondrial sequences in Agaricus bitorquis. Curr Genet 29: 370-376.

46. Robison MM, Royer JC, Horgen PA (1991). Homology between mitochondrial DNA of Agaricus bisporus and an internal portion of a linear mitochondrial plasmid of Agaricus bitorquis. Curr Genet 19: 495-502.

47. Bois F, Barroso G, Gonzalez P, Labarère J (1999) Molecular cloning, sequence and expression of Aa-polB, a mitochondrial gene encoding a family B DNA polymerase from the edible basidiomycete Agrocybe aegerita. Mol Gen Genet 261: 508-513.

48. Barroso G, Bois F, Labarère J (2001). Duplication of a truncated paralog of the family B DNA polymerase gene Aa-polB in the Agrocybe aegerita mitochondrial genome. Appl Environ Microbiol 67: 1739-1743.

49. Wang Y, Zeng F, Hon CG, Zhang Y, Leung FC (2008) The mitochondrial genome of the Basidiomycete fungus Pleurotus ostreatus (oyster mushroom). FEMS Microbiol Lett 280: 34-41.

50. Wibberg D, Jelonek L, Rupp O, Hennig M, Eikmeyer F, et al. (2012) Establishment and interpretation of the genome sequence of the phytopathogenic fungus Rhizoctonia solani AG1-IB isolate 7/3/14. J Biotechnol 167: 142155 . 